\title{
Antonio Cornejo Polar y la república mundial de las letras
}

\author{
Antonio Cornejo Polar And The World Republic Of Letters
}

\author{
Yolanda Westphalen Rodríguez \\ Universidad Nacional Mayor de San Marcos, Lima, Perú \\ Contacto: ywestphalenr@unmsm.edu.pe \\ https://orcid.org/0000-0003-2424-1899
}

\begin{abstract}
Resumen
El artículo analiza las categorías de totalidad contradictoria y heterogeneidad desarrolladas por Antonio Cornejo Polar, así como otras categorías postuladas desde el ámbito latinoamericano, para tratar de entender la dinámica de la mundialización del sistema literario, no como un proceso lineal de homogeneización, sino como un proceso caracterizado por la transculturación, el multilingüismo, el multiculturalismo, la hibridez y la heterogeneidad, realidad múltiple atravesada por complejos conflictos ideológicos e interculturales, es decir, no solo por la coexistencia en la diversidad, sino por el carácter conflictivo de este proceso. Se cuestiona, asimismo, el concepto de Franco Moretti de "sistema-mundo", y el planteamiento de Pascale Casanova sobre la República Mundial de las letras, por considerar que ambos planteamientos no logran romper con una perspectiva de análisis eurocéntrica.
\end{abstract}

Palabras clave: Totalidad contradictoria; Heterogeneidad; República mundial de las letras; Erocentrismo.

\begin{abstract}
The article analyze the categories of contradictory totality and heterogeneity developed by Antonio Cornejo Polar, as well as other postulated categories from the Latin American sphere, to try to understand the dynamics of the globalization of the literary system, not as a linear process of homogenization, but as a process characterized by transculturation, multilingualism, multiculturalism, hybridity and heterogeneity, multiple reality crossed by complex ideological and intercultural conflicts, that is, not only by the coexistence in diversity, but by the conflictive character of this process. It also questions Franco Moretti's concept of "world-system", and Pascale Casanova's approach to the World Republic of Letters, considering that both approaches fail to break with a perspective of Eurocentric analysis.
\end{abstract}

Keywords: Contradictory totality; Heterogeneity; World republic of letters; Eurocentrism. 
El objetivo de esta reflexión es relacionar las categorías de campo literario, sistema-mundo y república mundial de las letras desarrolladas por Pierre Bourdieu, Franco Moretti y Pascale Casanova, respectivamente, con las de totalidad contradictoria y heterogeneidad establecidas por Antonio Cornejo Polar, a fin de abordar la problemática del sistema literario mundial desde otra óptica.

La noción de campo, junto con las de habitus y capital, forma parte de los conceptos centrales de la obra del sociólogo francés Pierre Bourdieu ${ }^{1}$. Su formulación marcó un hito en la moderna disciplina de la sociología de la cultura. Para él, un campo es un sistema de relaciones sociales definido por la posesión y producción de una forma específica de capital. Se trata de un sistema de fuerzas en conflicto que se definen tanto por relaciones de competencia y complementariedad como por la posición que ocupan los miembros de este campo con relación a la estructura de poder de la sociedad en su conjunto.

En el caso del enfoque sociológico de la producción intelectual, hay que situar al artista y la obra dentro de un sistema de relaciones que incluye artistas, editores, críticos y públicos, los cuales determinan las condiciones específicas de producción y circulación de sus productos. A esto es lo que Bourdieu denomina campo cultural. En este campo, la lucha se da por la posesión y producción de cierto tipo de capital; capital que en el caso de la cultura, en general, y de la literatura, en particular, no es económico sino simbólico. Así,

El campo literario (etc.) es un campo de fuerzas que actúa sobre todos aquellos que entran en él, y de manera diferencial según la posición que ocupen a su interior (es decir, para considerar puntos muy distantes, el de autor de piezas de éxito o la de un poeta de vanguardia), al mismo tiempo que campos de competencia que tienden a conservar o transformar ese campo de fuerzas. (Bourdieu, 1991, pp. 4-5; traducción propia)

El campo cultural - y dentro de él, el literario - está dotado de una autonomía relativa que obedece a un proceso de autonomización que comienza con el surgimiento del capitalismo. Los agentes o sistemas de agentes definen su posición al interior de dicho campo, luchan y obtienen su legitimación cultural 
a partir de la dinámica interna del campo intelectual y de las reglas específicas del mismo. ¿Cuáles son estas reglas? ¿Quién le da valor a la obra? El valor de la obra o el problema de la legitimidad dependen de todo un sistema de relaciones sociales que el creador sostiene con el conjunto de los agentes al interior del mismo: críticos, artistas, públicos, editores. El análisis de dicho campo implica, por lo tanto, según Bourdieu, seguir tres pasos:

[...] primero, situar el campo literario (etc.) en el seno del campo de poder, con el cual se encuentra en relación de microcosmos a macrocosmos; en segundo lugar, analizar la estructura interna del campo literario (etc.), universo que obedece a sus propias leyes de funcionamiento y de transformación, es decir, a la estructura de relaciones objetivas entre las posiciones que ocupan en él individuos o grupos colocados en situación de competencia por la legitimidad artística; por último, analizar el habitus de los ocupantes de estas posiciones, es decir, los sistemas de las disposiciones que, siendo el producto de una trayectoria social y de una posición al interior del campo literario (etc.) encuentran en esta posición una ocasión más o menos favorable de actualizarse. (1991, pp. 5-6; traducción propia)

Sin embargo, el campo literario peruano y latinoamericano se formó también en una relación de unidad y conflicto con el campo literario mundial. Por ello es imprescindible examinar las categorías que se han creado para comprender este fenómeno. Pascale Casanova (2005) configura el concepto de "la literatura como mundo"; es decir, a diferencia de Bourdieu, ya no concibe la noción de campo literario en términos estrictamente nacionales, sino internacionales, ya que - según ella - no existe una coincidencia necesaria entre fronteras nacionales y fronteras del espacio literario. Cada escritor se define, en tal sentido, por una situación doble: según la posición que ocupa en el espacio nacional, y de acuerdo con su ubicación en el espacio internacional, instancias en interacción -léase, en lucha - en el mismo campo. Casanova trata de superar así las tendencias esencialistas en el ámbito de los estudios literarios que limitan sus análisis a una crítica literaria interna y a una ruptura total entre el texto y el mundo; ello con el fin de restablecer las relaciones entre literatura, historia y mundo, sin perder de vista la irreductible singularidad del texto. Dicha autora propone entonces la hipótesis de un espacio literario mundial en el que se expresarían las distintas 
luchas — políticas, sociales, nacionales, de género, étnicas—, pero “[...] de acuerdo con una lógica literaria y en formas literarias" (2005, p. 67), hipótesis que formula de la siguiente manera:

Por consiguiente, otro mundo, cuyas divisiones y fronteras son relativamente independientes de las fronteras políticas y lingüísticas. Y con sus propias leyes, su propia historia, sus revueltas y revoluciones específicas, un mercado en el que se comercie con valores no mercantiles, dentro de una economía no económica, y medido, como veremos, por una escala temporal estética. Este Mundo de las Letras funciona en su mayor parte de manera invisible, excepto para aquellos que pueden ver con más claridad que los otros las formas de violencia y dominación que operan en su interior.

Llamamos a esta área intermedia el "espacio literario mundial". No es más que una herramienta que debería ponerse a prueba mediante la investigación concreta, un instrumento que podría proporcionar una explicación sobre la lógica y la historia de la literatura, sin caer en la trampa de la autonomía total. (Casanova, 2005, p. 67)

Ahora bien, según Casanova, en este campo literario internacional existe una idea única de "modernidad estética" que opera como un meridiano de Greenwich (medida de tiempo literario). Este nos permite calcular la distancia entre el centro y los protagonistas del espacio literario y establecer qué obras son aceptadas y legitimadas como modernas y cuáles no:

Otro indicador — más difícil de observar- es la aparición de una medición específica del tiempo, común para todos los actores. Cada nuevo miembro debe reconocer de partida un punto de referencia, una norma respecto a la cual lo vayan a medir; todas las posiciones están localizadas en referencia a un centro en el que se determina el presente literario. Propongo llamar a este centro "Meridiano de Greenwich" de la literatura. Al igual que la línea imaginaria, arbitrariamente escogida para determinar las líneas de longitud, contribuye a la organización real del mundo y permite medir distancias y evaluar posiciones en la superficie del planeta, también el meridiano literario nos permite calcular la distancia entre el centro y los protagonistas dentro del espacio literario. Es el lugar en el que la medición del tiempo literario - es decir la evaluación de la modernidad estética - se cristaliza, se disputa y se elabora. Lo considerado moderno aquí, en un momento dado será considerado el "presente": textos que "dejarán su huella", capaces de modificar las actuales normas estéticas. Estas obras 
servirán, durante un tiempo al menos, de unidades de medición dentro de una cronología específica, modelos de comparación para producciones posteriores. (Casanova, 2005, pp. 69-70)

Franco Moretti, por su parte, sostiene la teoría del "sistema-mundo" $(2000 ; 2003)$. Con ella afirma la unidad y desigualdad del sistema literario y su oposición entre centro y periferia. He tomado ambas posturas porque concuerdo con la existencia del campo literario y de un sistema literario mundial, y porque considero que el concepto de una república internacional de las letras permite esclarecer la naturaleza de la lucha de los escritores en el Perú y América Latina por ocupar un espacio, tanto local como dentro del movimiento literario internacional.

Considero, sin embargo, que plantear la problemática desde el punto de vista de las categorías de totalidad contradictoria y de heterogeneidad desarrolladas por Antonio Cornejo Polar, y estudiadas posteriormente por un conjunto de teóricos y críticos latinoamericanos, particularmente por Carlos

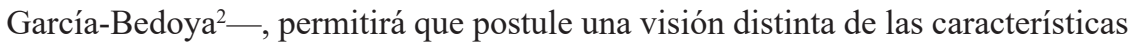
del campo literario peruano y latinoamericano, así como de la relación entre dichos campos y el internacional.

Antonio Cornejo Polar propone dos categorías que permiten una visión de conjunto de los procesos históricos, pero lo hace desde una perspectiva que renueva la historiografía literaria clásica. En su famoso discurso de ingreso a la Academia Peruana de la Lengua, afirma:

Interesa, entonces, adoptar una perspectiva y articular las categorías teóricas con conocimientos históricos. Se burlan así los riesgos de la falsa neutralidad, pues asumir un tiempo es asumir también su conflictividad social, a la par que se alejan los peligros del idealismo y del empirismo, peligros que, tratándose del estudio de una literatura nacional, implican en el primer caso la esencialización de sus dos términos, como si la literatura no fuera cambiante y la nación una fluencia continua, y en el segundo, la simple recopilación de datos sin sentido orgánico ni procesal. Es en el espacio formado por la relación dialéctica entre teoría e historia donde debe fundarse una nueva concepción de la literatura peruana. A colaborar en este esfuerzo, 
que sin duda tendrá que ser colectivo, están destinadas las siguientes reflexiones. (1983, p. 38)

El gran reto colectivo que Cornejo plantea es articular las categorías teóricas, como las de totalidad contradictoria y heterogeneidad, y comprenderlas en su desarrollo histórico. Para él, el espacio de la literatura nacional y del campo literario peruano es un espacio conflictivo en el que se han desarrollado diversos sistemas literarios y lecturas críticas de dicho proceso. Alude, en tal sentido, a la concepción de la literatura peruana como hispánica y al ideal de una nación unitaria y homogénea de raíces ibéricas propugnado por los principales representantes de la corriente hispanista (De la Riva-Agüero, 1905); examina la postura de la literatura mestiza, categoría tras la cual se esconden posiciones disímiles, dependiendo del grado de influencia que tuvieran los componentes de dicho "mestizaje”, mayor presencia hispánica (Gálvez, 1915), predominio de la cultura indígena (More, 1924) o un "peruanismo totalista" basado en una síntesis armónica (Sánchez, 2001).

Reconoce Cornejo, sin embargo, que si bien la categoría del mestizaje no rompe teóricamente con la perspectiva hispanista ni combate sus perspectivas epistemológicas, históricamente permitió que se incluyera a la literatura prehispánica como la primera etapa de la literatura. Los límites de este aporte estuvieron en la aceptación implícita de que la producción literaria en lenguas nativas había concluido con la Conquista, por lo que las manifestaciones posteriores no eran consideradas o se las remitía al campo del folklore. Plantea, por último, la crisis de la categoría de la unidad para comprender la multiplicidad de los sistemas literarios que se dan en el Perú: el culto, el popular y el producido en lenguas indígenas. Al reivindicar la perspectiva de Mariátegui del carácter no orgánicamente nacional de la literatura peruana y la periodización de su historia como la de un proceso de lucha entre campos y fuerzas en conflicto, Cornejo llega a la formulación de la categoría de la unidad contradictoria y conflictiva de sistemas literarios en el Perú; así, estos solo pueden ser explicados como parte del proceso histórico general de la sociedad. No se trata de la categoría de la heterogeneidad 
como sinónimo de pluralidad, es decir de la coexistencia armónica de diversos sistemas literarios y fuerzas al interior del campo, sino de la naturaleza conflictiva de dicho proceso y la necesidad de aprehenderlo como totalidad.

Postulamos, entonces, que estas categorías nos permiten abordar también la concepción de la república mundial de las letras como una totalidad multicéntrica, conflictiva y heterogénea que rompe con la concepción eurocéntrica dominante con la que se define actualmente dicho campo. Tanto el concepto de Franco Moretti de "sistema-mundo" y la relación centro-periferia como el planteamiento de Pascale Casanova (2005, pp. 71-90) sobre la república mundial de las letras, regida por un meridiano estético y una relación entre dominantes y dominados, mantienen una posición eurocéntrica. Si bien ambos autores señalan que este espacio literario internacional no es una esfera desnacionalizada establecida por encima de todas las demás, sino que todos los habitantes de dicho espacio son parte de él, añaden que cada uno de ellos tiene que definir su situación en función de la doble posición que ocupan, tanto en el espacio nacional como de acuerdo con el lugar que este espacio nacional ocupa en el mundial. Y a nuestro parecer esta es la formulación problemática, porque al enunciar su discurso ellos estarían otorgándole una posición jerárquica a la producción de la literatura europea, concebida como el parámetro de lo universal.

Para Moretti, las literaturas dominadas o de la periferia surgen del encuentro entre la realidad occidental y la realidad local. Así, para ejemplificar ello, estudia a la novela como la forma literaria más extendida y las variaciones locales a esta forma. Para Casanova se trata de un espacio jerarquizado y desigual, regido por valores propios de prestigio, atribuidos a las creaciones literarias inestimables, asignadas por ella a las literaturas más antiguas, que serían las más autónomas y las más dotadas de herencia literaria y de recursos, es decir los espacios europeos. No se trata tan solo de que en su análisis no hayan visto particularidades de otros espacios, como la importancia del desarrollo de la poesía en América Latina, objeción correcta planteada por Efraín Kristal a Moretti (Kristal, 2002), sino de los presupuestos mismos sobre los que se erigen sus planteamientos. 
Cuestionamos, asimismo, la idea de una modernidad estética única, pues a diferencia de lo sostenido por la crítica literaria francesa Casanova, no consideramos que haya un "meridiano de Greenwich" que permita regionalizar lo moderno para atribuirlo como aquello dado en territorio europeo, debido a que esta conquista puede realizarse desde un lugar de enunciación americano. Dicha línea imaginaria para establecer el centro de la medida del tiempo literario (o meridiano de Greenwich) se convierte en una suerte de cámara fija, que desde una posición de picado enfoca el campo literario internacional. Ni siquiera intenta incorporar las conquistas del multiperspectivismo y del simultaneísmo de las vanguardias, $\mathrm{u}$ otros tipos de tomas del lenguaje cinematográfico como el paneo para aprehender las múltiples facetas y temporalidades de este proceso. En realidad, la percepción del capital simbólico acumulado varía desde el punto de vista de los parámetros con los que se lo define, así si se privilegia la lengua escrita, si se aceptan como géneros solo aquellos que son dominantes de acuerdo con la tradición occidental, por ejemplo, la novela, y no se considera siquiera como posible género el huayno, género lírico íntimamente vinculado a la música, planteado por Arguedas. Es obvio, entonces, que los que ocupan una posición dominante en el campo literario internacional son los que van a decidir qué se considera el capital simbólico y qué no, y, por consiguiente, quién lo posee y quién no.

El problema es cómo se concibe el espacio de la literatura mundial: si se enuncia el discurso asumiendo que existe un centro, un meridiano, un espacio con mayor capital, en última instancia, un universal que se opone al particular de la periferia, del subordinado o del subalterno, o si se considera este planteamiento como una perspectiva de comparatismo jerarquizante y se rechaza que se atribuya a algunos las características de universal y a los otros de particular. Nuestro estudio se plantea la hipótesis de un espacio de la literatura mundial heterogéneo, desigual y en el que coexisten distintas temporalidades históricas y modernidades; en tal sentido, estimamos que el espacio universal de la literatura mundial solo puede ser concebido como un espacio concreto si se lo ve como la síntesis de múltiples manifestaciones literarias particulares, es decir, la unidad de la diversidad de campos literarios, capitales simbólicos y manifestaciones literarias. 
Desde esta perspectiva multicéntrica es que proponemos revisar la historia del campo literario peruano y latinoamericano, así como su relación con el espacio europeo. El enfoque propuesto cuestiona el que se le otorgue un lugar de privilegio a la literatura europea y se conviertan las características de una de las formas literarias particulares e históricamente determinadas en el punto de vista universal, porque consideramos que así no se puede aprehender la real naturaleza universal del fenómeno literario ni de la modernidad estética. Unidad conflictiva en lucha entre distintas formaciones socioculturales, tradiciones, géneros, formas de relacionarse entre sí y con el poder. El universal es la existencia de formas artísticas de expresión a través del arte verbal a escala mundial, pero las formas de hacerlo corresponden a diferentes universos socioculturales atravesados por sus propios conflictos políticos, sociales, lingüísticos, culturales, de clase, de género, de etnia, así como entre naciones. Es un universal desigual, sí, pero no en el sentido de naciones emergentes que avanzan hacia una concepción modélica de formas literarias, es un universal que contiene en sí varias formas particulares de definir la literatura, que asume como miembros a distintos tipos de personas, que se expresa en múltiples lenguas y se orienta a públicos disímiles, que opta por géneros discursivos distintos y está atravesado por distintas temporalidades históricas, que sin embargo coexisten.

Desde el ámbito latinoamericano se ha desarrollado una serie de categorías para tratar de entender la dinámica de la mundialización, no como un proceso lineal de homogeneización, sino como un desarrollo caracterizado por la hibridez, la heterogeneidad, el multilingüismo y el multiculturalismo; realidad múltiple atravesada por complejos conflictos ideológicos e interculturales, es decir, no solo por la coexistencia en la diversidad, sino por el carácter conflictivo de este proceso. Las categorías latinoamericanas que García-Bedoya considera tienen un gran potencial heurístico para comprender este proceso de la mundialización de la literatura son la transculturación planteada por Ángel Rama, el carácter híbrido de las culturas latinoamericanas propuesto por Néstor García Canclini y las categorías de totalidad contradictoria, de heterogeneidad y de sujeto migrante de Antonio Cornejo Polar: 
Las categorías introducidas por Rama, Cornejo Polar y García Canclini nos permiten acercarnos a una compleja problemática cuyas repercusiones trascienden las fronteras latinoamericanas y coinciden en la necesidad de diseñar una modernidad propia y no conformarse con una modernidad copia. Ya sea desde la perspectiva más específica de las literaturas ilustradas en diálogo con las culturas subalternas, o desde la más abarcadora de las múltiples manifestaciones artísticas y el campo general de la cultura, las categorías que introducen resultan altamente productivas. (García-Bedoya, 2011, p. 20)

Desde mi punto de vista, comprender la dinámica del desarrollo de la literatura y los campos literarios en Europa o en América Latina implica desechar cualquier concepción jerárquica sobre la existencia de literaturas mayores y menores. El estudio de la literatura peruana, latinoamericana e internacional supone comprender el espacio de la literatura mundial y la modernidad estética no como determinado a partir de espacios geográficos opuestos entre sí, a los que corresponderían espacios simbólicos desiguales entre dominantes y dominados, sino advertir que se trata de espacios simbólicos superpuestos, híbridos y/o diferenciados que combinan distintas temporalidades históricas, tradiciones y cronotopos, que existen y están en lucha entre sí, tanto a escala nacional como mundial. La modernidad de las manifestaciones estéticas contemporáneas de la literatura peruana y latinoamericana corresponde a una lógica y formas literarias específicas; puede no tener las mismas características de la modernidad europea, pero es una forma histórica y socialmente determinada de modernidad, igualmente válida que aquella. La perspectiva epistemológica de una razón dialógica intercultural — planteada por García-Bedoya - es una propuesta que debe permitir debatir — desde otra perspectiva — los problemas de la modernidad y de la mundialización de la literatura. Solo cabe destacar que la asunción de categorías es parte de la lucha simbólica al interior del campo literario mundial, y no es ni mucho menos neutra.

\section{Notas}

1 Revisar, al respecto, las siguientes obras de Pierre Bourdieu: "L'invention de la vie d'artiste" (1975); "L'illusion biographique" (1986); "El campo literario. Prerrequisitos críticos y principios de método" (1990); "Le champ littéraire" (1991); Las reglas 
del arte. Génesis y estructura del campo literario (1995); Campo de poder, campo intelectual. Itinerario de un concepto (2002); "Les apparatchiks de la recherche" (2002); y Bourdieu, Pierre y otros. "Du bon usage de l'ethnologie" (2003).

2 Véanse los siguientes textos de Carlos García-Bedoya: Para una periodización de la literatura peruana (1990); "Transculturación, heterogeneidad, hibridez: algunas reflexiones" (1998); "Los estudios culturales en debate: una mirada desde América Latina" (2001); "Categorías latinoamericanas para una mundialización intercultural" (2011); e Indagaciones heterogéneas (2012).

\section{Referencias bibliográficas}

Bourdieu, P. (1975). L'invention de la vie d'artiste. En Actes de la recherche en sciences sociales, 1(2), 67-93. doi: 10.3406/arss.1975.2458.

Bourdieu, P. (1986). L'illusion biographique. Actes de la recherche en sciences sociales, 62-63, 69-72. doi: 10.3406/arss.1986.2317.

Bourdieu, P. (1989-1990). El campo literario. Prerrequisitos críticos y principios de método. Criterios, (25-28), 20-42.

Bourdieu, P. (1991). Le champ littéraire. Actes de la recherche en sciences sociales, 89, 3-46. doi: 10.3406/arss.1991.2986.

Bourdieu, P. (1995). Las reglas del arte. Génesis y estructura del campo literario. Barcelona: Anagrama.

Bourdieu, P. (2002a). Campo de poder, campo intelectual. Itinerario de un concepto. Tucumán: Montressor.

Bourdieu, P. (2002b). Les apparatchiks de la recherche. Actes de la recherche en sciences sociales, 141-142, 82-83. doi: 10.3406/arss.2002.3519.

Bourdieu, P., et al. (2003). Du bon usage de l'ethnologie. Actes de la recherche en sciences sociales 150, 9-18. doi:10.3406/arss.2003.2767.

Casanova, P. (2005). La literatura como mundo. New Left Revie, 31, 66-83. Recuperado de http://newleftreview.es/authors/pascale-casanova.

Cornejo Polar, A. (1983). La literatura peruana: totalidad contradictoria. Revista de Crítica Literaria Latinoamericana, 9(18), 37-50.

De la Riva Agüero y Osma, J. (1905). Carácter de la Literatura del Perú Independiente. Lima: Librería Francesa científica Galland. 
Gálvez, J. (1915). Posibilidad de una genuina literatura nacional. Lima: Casa Editora M. Moral.

García-Bedoya, C. (1990). Para una periodización de la literatura peruana. Lima: Latinoamericana Editores.

García-Bedoya, C. (1998). Transculturación, heterogeneidad, hibridez: algunas reflexiones. En T. Escajadillo (Ed.), Perfil y entraña de Antonio Cornejo Polar (79-87). Lima: Amaru Editores.

García-Bedoya, C. (2001). Los estudios culturales en debate: una mirada desde América Latina. Revista de Crítica Literaria Latinoamericana, 27(54), 195-211.

García-Bedoya, C. (2011). Categorías latinoamericanas para una mundialización intercultural. Cuadernos Inter-c-a-mbio, 8(9), 15-37.

García-Bedoya, C. (2012). Indagaciones heterogéneas. Lima: Grupo Pakarina.

Kristal, E. (2002). “Considerando en frío...”. Respuesta a Franco Moretti. New Left Review, 15, 56-68. Recuperado de http://newleftreview.es/authors/ efrain-kristal.

More, F. (1924). De un ensayo acerca de las literaturas del Perú. El Diario de la Marina de La Habana (también en El Norte de Trujillo, el mismo año).

Moretti, F. (2000). Conjeturas sobre la literatura mundial. New Left Review, 3, 65 76. Recuperado de http://newleftreview.es/authors/franco-moretti.

Moretti, F. (2003). Más conjeturas sobre la literatura mundial. New Left Review, 20, 83-91. Recuperado de http://newleftreview.es/authors/francomoretti.

Sánchez, L. A. (2001). Nosotros: ensayo sobre una literatura nacional. En Cien años de Luis Alberto Sánchez. Homenaje del Congreso de la República (115-142). Lima: Fondo Editorial del Congreso del Perú. 